\title{
385.
}

\section{ON THE CORRESPONDENCE OF TWO POINTS ON A CURVE.}

[From the Proceedings of the London Mathematical Society, vol. I. (1865-1866), No. viI. pp. 1-7. Read April 16, 1866.]

1. IN a unicursal curve the coordinates $(x, y, z)$ of any point of the curve are proportional to rational and integral functions of a variable parameter $\theta$. Hence, if two points of the curve correspond in suchwise that to a given position of the first point there correspond $\alpha^{\prime}$ positions of the second point, and to a given position of the second point $\alpha$ positions of the first point, the number of points which correspond each to itself is $=\alpha+\alpha^{\prime}$. For let the two points be determined by their parameters $\theta, \theta^{\prime}$ respectively-then to a given value of $\theta$ there correspond $\alpha^{\prime}$ values of $\theta^{\prime}$, and to a given value of $\theta^{\prime}$ there correspond $\alpha$ values of $\theta$; hence the relation between $\left(\theta, \theta^{\prime}\right)$ is of the form $(\theta, 1)^{\alpha}\left(\theta^{\prime}, 1\right)^{\alpha^{\prime}}=0$; and writing therein $\theta^{\prime}=\theta$, then for the points which correspond each to itself, we have an equation $(\theta, 1)^{\alpha+\alpha^{\prime}}=0$ of the order $\alpha+\alpha^{\prime}$; that is, the number of these points is $=\alpha+\alpha^{\prime}$.

2. Hence for a unicursal curve we have a theorem similar to that of M. Chasles' for a line, viz., the theorem may be thus stated:

If two points of a unicursal curve have an $\left(\alpha, \alpha^{\prime}\right)$ correspondence, the number of united points is $=\alpha+\alpha^{\prime}$.

3. But a unicursal curve is nothing else than a curve with a deficiency $D=0$, and we thence infer

Theorem. If two points of a curve with deficiency $D$ have an $\left(\alpha, \alpha^{\prime}\right)$ correspondence, the number of united points is $=\alpha+\alpha^{\prime}+2 k D$; in which theorem $2 k$ is a coefficient to be determined.

4. Suppose that the corresponding points are $P, P^{\prime}$ and imagine that when $P$ is given, the corresponding points $P^{\prime}$ are the intersections of the given curve by a c. VI. 
curve $\Theta$ (the equation of the curve $\Theta$ will of course contain the coordinates of $P$ as parameters, for otherwise the position of $P^{\prime}$ would not depend upon that of $P$ ). I find that if the curve $\Theta$ has with the given curve $k$ intersections at the point $P$, then in the system of $\left(P, P^{\prime}\right)$, the number of united points is

$$
\mathrm{a}=\alpha+\alpha^{\prime}+2 k D
$$

whence in particular, if the curve $\Theta$ does not pass through the point $P$, then the number of united points is $=\alpha+\alpha^{\prime}$, as in a unicursal curve.

4*. The foregoing theorem is easily proved in the particular case where the $k$ intersections at the point $P$ take place in consequence of the curve $\theta$ having a $k$-tuple point at $P$. Taking $U=(x, y, z)^{m}=0$ as the equation of the given curve (which for greater simplicity is assumed to be a curve without singularities), then if we suppose $(x, y, z)$ to be the coordinates of the point $P$, and $\left(x^{\prime}, y^{\prime}, z^{\prime}\right)$ to be the coordinates of the point $P^{\prime}$, write $U=(x, y, z)^{m}, U^{\prime}=\left(x^{\prime}, y^{\prime}, z^{\prime}\right)^{m}, U^{\prime}$ being what $U$ becomes on writing therein $\left(x^{\prime}, y^{\prime}, z^{\prime}\right)$ in place of $(x, y, z)$; and

$$
\Theta=(x, y, z)^{a}\left(x^{\prime}, y^{\prime}, z^{\prime}\right)^{\alpha^{\prime}}\left(y z^{\prime}-y^{\prime} z, z x^{\prime}-z^{\prime} x, x y^{\prime}-x^{\prime} y\right)^{k},
$$

viz., $\Theta$ is a function of the order $k$ in $y z^{\prime}-y z^{\prime}, z x^{\prime}-z^{\prime} x, x^{\prime} y-x^{\prime} y$, the coefficients of the several powers and products of these quantities being functions of the order $a$ in $(x, y, z)$ and of the order $a^{\prime}$ in $\left(x^{\prime}, y^{\prime}, z^{\prime}\right)$, which functions are such that they do not all of them vanish, identically, or in virtue of the equation $U=0$, on writing therein $\left(x^{\prime}, y^{\prime}, z^{\prime}\right)=(x, y, z)$. Taking for a moment $(x, y, z)$ as current coordinates, suppose that the equation of the given curve is $U=0$; then if $(x, y, z)$ are the coordinates of the point $P$, we have $U=0$, and similarly if $\left(x^{\prime}, y^{\prime}, z^{\prime}\right)$ are the coordinates of the point $P^{\prime}$ we have $U^{\prime}=0$. The equation $\Theta=0$, considering therein $(x, y, z)$ as the coordinates of the given point $P$ (and so as parameters satisfying the equation $U=0$ ) and $\left(x^{\prime}, y^{\prime}, z^{\prime}\right)$ as current coordinates, will be a curve having a $k$-tuple point at $P$, we have thus the case above supposed; and $P$ being given, the corresponding points $P^{\prime}$ are given as the intersections of the curves $U^{\prime}=0, \Theta=0$, which are respectively of the orders $m$ and $a^{\prime}+k$; the total number of intersections is thus $=m\left(a^{\prime}+k\right)$, but inasmuch as the curve $\Theta=0$ has a $k$-tuple point at $P, k$ of these intersections coincide with the point $P$, and the number of the remaining intersections, that is the number of positions of the point $P^{\prime}$, is $=m a^{\prime}+(m-1) k$. Similarly when $P^{\prime}$ is given, the number of positions of the point $P$ is $=m a+(m-1) k$ : and we have therefore

$$
\alpha+\alpha^{\prime}=m\left(a+a^{\prime}\right)+2(m-1) k .
$$

To find the united points, it is to be observed, that upon writing $\left(x^{\prime}, y^{\prime}, z^{\prime}\right)=(x, y, z)$, the function $\Theta$ becomes identically $=0$; but if we suppose, in the first instance, that $P^{\prime}, P$, are consecutive points on the curve $U=0$, then we have

$$
y z^{\prime}-y^{\prime} z: z x^{\prime}-z^{\prime} x: x y^{\prime}-x^{\prime} y=\delta_{x} U ; \delta_{y} U ; \delta_{z} U ;
$$

and the equation $\Theta=0$ assumes the form

$$
\Theta=(x, y, z)^{a}(x, y, z)^{a^{\prime}}\left(\delta_{x} U, \delta_{y} U, \delta_{z} U\right)^{k}=0,
$$


which, $(x, y, z)$ being current coordinates, is the equation of a curve of the order $a+a^{\prime}+(m-1) k$; the united points are the intersection of this curve with the given curve $U=0$, and the number of the united points is thus

$$
\mathrm{a}=m\left(\alpha+\alpha^{\prime}\right)+m(m-1) k .
$$

Hence attending to the above-mentioned value of $\alpha+\alpha^{\prime}$, we have

$$
\mathrm{a}=\alpha+\alpha^{\prime}+(m-1)(m-2) k .
$$

But in the case of a curve $U=0$, without singularities, we have $2 D=(m-1)(m-2)$, and we have thus the required formula

$$
\mathrm{a}=\alpha+\alpha^{\prime}+2 k D \text {. }
$$

The investigation in the case where the $k$ intersections at $P$ arise wholly or in part from a contact of the curve $\Theta$, or any branch or branches thereof, with the given curve $U$, is more difficult, and $I$ abstain from entering upon it.

I apply the theorem to some examples:

5. Investigation of the class of a curve of the order $m$ with $\delta$ dps. Take as corresponding points on the curve two points, such that the line joining them passes through a fixed point $O$ : the united points will be the points of contact of the tangents through $O$; that is, the number of the united points is equal to the class of the curve. The curve $\Theta$ is here the line $O P$, which has with the given curve a single intersection at $P$; that is, we have $k=1$. The points $P^{\prime}$ corresponding to a given position of $P$ are the remaining $m-1$ intersections of $O P$ with the curve; that is, we have $\alpha^{\prime}=m-1$; and in like manner $a=m-1$. Hence the class is $=2(m-1)+2 D$, viz., this is $=(2 m-2)+\left(m^{2}-3 m+2-2 \delta\right)$, which is $=m^{2}-m-2 \delta$, as it should be.

6. It is in the foregoing example assumed that the dps are none of them cusps; if the curve has $\delta+\kappa \mathrm{dps}, \kappa$ of which are cusps (or what is the same thing, $\delta$ nodes and $\kappa$ cusps); then the number of united points is equal $2(m-1)+2 D,=m^{2}-m-2 \delta-2 \kappa$; but in this case each of the cusps is reckoned as a united point, and we have, therefore, class $+\kappa=m^{2}-m-2 \delta-2 \kappa$, that is, class $=m^{2}-m-2 \delta-3 \kappa$. This will serve as an instance of the special considerations which are required in the case of a curve with cusps, but in what follows, I shall assume that the dps are none of them cusps and thus attend to the case of a curve of the order $m$, with $\delta \mathrm{dps}$, and therefore of the class $n=m^{2}-m-2 \delta$, and of the deficiency $D=\frac{1}{2}(m-1)(m-2)-\delta,=\frac{1}{2}(n-2 m+2)$.

7. Investigation of the number of inflexions. Taking the point $P^{\prime}$ to be a tangential of $P$ (that is, an intersection of the curve by the tangent at $P$ ), the united points are the inflexions, and the number of the united points is equal to the number of inflexions. The curve $\Theta$ is here the tangent at $P$, having with the given curve two intersections at $P$; that is $k=2 . P^{\prime}$ is any one of the $m-2$ tangentials of $P$, hence $\alpha^{\prime}=m-2$; and $P$ is the point of contact of any one of the $n-2$ 
tangents from $P^{\prime}$ to the curve, that is, $\alpha=n-2$. Hence the number of inflexions is $=(m-2)+(n-2)+4 D,=m+n-4+2(n-2 m+2),=3(n-m)$, which is right.

8. For the purpose of the next example it is necessary to present the fundamental equation $\mathrm{a}=\alpha+\alpha^{\prime}+2 k D$ under a more general form. The curve $\Theta$ may intersect the given curve in a system of points $P^{\prime}$ each $p$ times, a system of points $Q^{\prime}$ each $q$ times, \&c., in such manner that the points $\left(P, P^{\prime}\right)$, the points $\left(P, Q^{\prime}\right)$, \&c., are pairs of points corresponding to each other according to distinct laws; and we shall then have the numbers $\left(a, \alpha, \alpha^{\prime}\right),\left(b, \beta, \beta^{\prime}\right)$, \&c., belonging to these pairs respectively; viz. $\left(P, P^{\prime}\right)$ are points having an $\left(\alpha, \alpha^{\prime}\right)$ correspondence, and the number of united points is $=\mathrm{a}$; similarly $\left(P, Q^{\prime}\right)$ are points having a $\left(\beta, \beta^{\prime}\right)$ correspondence, and the number of united points is $=b$; and so on. The theorem then is

$$
p\left(a-\alpha-\alpha^{\prime}\right)+q\left(b-\beta-\beta^{\prime}\right)+\ldots=2 k D .
$$

9. Investigation of the number of double tangents:-Take $P^{\prime}$, an intersection with the curve of a tangent drawn from $P$ to the curve (or what is the same thing, $P, P^{\prime}$ cotangentials of any point of the curve); the united points are here the points of contact of the several double tangents of the curve; or if $\tau$ be the number of double tangents, then the number of united points is $=2 \tau$. The curve $\Theta$ is the system of the $n-2$ tangents from $P$ to the curve; each tangent has with the curve 1 intersection at $P$, that is, $k=n-2$; each tangent, besides, meets the curve in the point of contact $Q^{\prime}$ twice, and in $(m-3)$ points $P^{\prime}$. Hence, if (a, $\left.\alpha, \alpha^{\prime}\right)$ refer to the points $\left(P, Q^{\prime}\right)$, and $\left(2 \tau, \beta, \beta^{\prime}\right)$ to the points $\left(P, P^{\prime}\right)$, we have

$$
2\left(a-\alpha-\alpha^{\prime}\right)+2 \tau-\beta-\beta^{\prime}=2(n-2) D .
$$

Moreover, from the last example the value of $a-\alpha-\alpha^{\prime}$ is $=4 D$, and the formula thus becomes

$$
2 \tau-\beta-\beta^{\prime}=2(n-6) D
$$

but from above it appears that we have $\beta=\beta^{\prime}=(n-2)(m-3)$, whence

$$
\begin{aligned}
2 \tau & =2(n-2)(m-3)+2(n-6) D, \\
& =2(n-2)(m-3)+(n-6)(n-2 m+2), \\
& =n^{2}-10 n+8 m,
\end{aligned}
$$

which is right; in fact, observing that $\iota$ (the number of inflexions) is $=3 n-3 m$, the formula is equivalent to $2 \tau+3 \iota=n^{2}-n-m$, that is, $m=n^{2}-n-2 \tau-3 \iota$.

In the foregoing examples the curve $\Theta$ is a line or system of lines; but I give an example in which $\Theta$ is a system of conics, and in which, as will appear, we have to consider the two characteristics $(\mu, \nu)$ of the system.

10. Investigation of the number of conics which can be drawn, satisfying any four conditions, and touching the given curve; or say of the number of the conics (4Z) (1). Take $P^{\prime}$, an intersection of the given curve by a conic $(4 Z)$ passing through the point $P$, then the number of the united points is equal to that of the conics $(4 Z)(1)$. The curve $\Theta$ is here the system of the conics $(4 Z)$ which pass through $P$; 
hence, if $(\mu, \nu)$ be the characteristics of the system of conics $(4 Z)$, the number of the conics through $P$ is $=\mu$; each of these has with the given curve 1 intersection at $P$, and consequently $k=\mu$. Moreover, each of the conics besides meets the curve in $(2 m-1)$ points, and consequently $\alpha=\alpha^{\prime}=\mu(2 m-1)$. Hence the formula gives the number of united points

or, as this may be written,

$$
\begin{aligned}
& =2 \mu(2 m-1)+\mu(n-2 m+2), \\
& =\mu(n+2 m) ;
\end{aligned}
$$

$$
=\mu n+\nu m+m(2 \mu-\nu) .
$$

But the system of conics (4Z) contains $(2 \mu-\nu)$ point-pairs (coniques infiniment aplaties), each of which, regarded as a pair of coincident lines, meets the given curve in $m$ pairs of coincident points; that is, the point-pair is to be considered as a conic touching the given curve in $m$ points; and there is on this account a reduction $=m(2 \mu-\nu)$ in the number of the united points; whence, finally, the number of the conics $(4 Z)(1)$ is $=\mu n+\nu m$. It is hardly necessary to remark that it is assumed that the conditions $(4 Z)$ are conditions having no special relation to the given curve.

11. As a final example, suppose that the point $P$ on a given curve of the order $m$, and the point $Q$ on a given curve of the order $m^{\prime}$, have an $\left(\alpha, \alpha^{\prime}\right)$ correspondence, and let it be required to find the class of the curve enveloped by the line $P Q$. Take an arbitrary point $O$, join $O Q$, and let this meet the curve $m$ in $P^{\prime}$, then $\left(P, P^{\prime}\right)$ are points on the curve $m$, having a $\left(m^{\prime} \alpha, m x^{\prime}\right)$ correspondence; in fact, to a given position of $P$ there correspond $\alpha^{\prime}$ positions of $Q$, and to each of these $m$ positions of $P^{\prime}$, that is, to each position of $P$ there correspond $m \alpha^{\prime}$ positions of $P^{\prime}$; and similarly to each position of $P^{\prime}$ there correspond $m^{\prime} \alpha$ positions of $P$. The curve $\Theta$ is the system of the lines drawn from each of the $\alpha^{\prime}$ positions of $Q$ to the point $O$, hence the curve $\Theta$ does not pass through $P$, and we have $k=0$. Hence the number of the united points $\left(P, P^{\prime}\right)$, that is, the number of the lines $P Q$ which pass through the point $O$, is $=m \alpha^{\prime}+m^{\prime} \alpha$, or this is the class of the curve enveloped by $P Q$.

12. It may be remarked, that if the two curves are curves in space (plane or of double curvature), then the like reasoning shows that the number of the lines $P Q$ which meet a given line $O$ is $=m \alpha^{\prime}+m^{\prime} \alpha$, that is, the order of the scroll generated by the line $P Q$ is $=m \alpha^{\prime}+m^{\prime} \alpha$. 